\title{
Neural network adaptive real-time optimizing control of industrial processes
}

\author{
Normah Abdullah ${ }^{1}$, Muhammad Anas Mohd Razali', Mohammed Hamood Othman Ahmed ${ }^{3}$, Mohd Zaki \\ Nuawi $^{4}$, Mohd Marzuki Mustafa $^{5}$, Zulkifli Mohd Nopiah ${ }^{6}$, Azah Mohamed ${ }^{7}$, Abu Bakar Mohamad
}

${ }^{1,2,3,8}$ Department of Chemical and Process Engineering, ${ }^{4}$ Department of Mechanical and Materials Engineering, ${ }^{5,7}$ Department of Electrical, Electronics and System Engineering, ${ }^{6}$ Fundamental Studies of Engineering Unit, Faculty of Engineering and Built Environment, National University of Malaysia, 43600 Bangi, Selangor, Malaysia. Article Info: Submitted on March 20, 2017, Accepted on June 20, 2017.

\begin{abstract}
Real-time optimization (RTO) has attracted considerable interest among researchers and industries for being able to optimise the plant economics such as product efficiency, product quality and process safety in the wake of increasing global competitions. The success of RTO depends much on the quality of model being used in the optimisation. The present study was carried out to explore the use of artificial neural network (ANN) to improve the quality of the model being used in the modified two step (MTS) technique. The MTS is a real-time optimising control algorithm of the modifier adaptation scheme which is used to determine the optimum steadystate control set-points. The proposed new version of MTS technique will be using process model based on ANN. A laboratory scale process of a two continuous stirred tank heat exchanger in series (2CSTHEs) is used as a case study. The multilayer feed forward ANN architecture 4-10-6 with linear function was used to model the 2CSTHEs and then integrates into the MTS technique, the resulted algorithm will be known as Iterative Neural Network Modified Two Step (INNMTS) technique. Simulation studies were conducted to test the performance of the INNMTS technique on the 2CSTHEs process. The results show that the overall value for the coefficient of determination $\left(R^{2}\right)$ is equal to one, which indicates adequacy of the model proposed for the prediction of the behavior of 2CSTHEs system. When NN model of 2CSTHEs is applied to the INNMTS technique, the model-plant mismatch is greatly reduced to almost zero, which indicates by significant reduction in the number of iterations to 5 which requires by INNMTS compared to 16 iterations by the MTS technique to converge to optimal real solution.
\end{abstract}

Keywords:Real-time optimisation, Artificial neural network, Modified two step technique, Process modelling.

\section{Introduction}

In large-scale industrial processes for determining optimal operating conditions are typically addressed by process control activities which are organised in the form of a hierarchy with required functions at the lower levels and desired, but optimal, functions at the higher levels. During plant operation, the optimum operating conditions is recalculated on the regular basis at control level activity known as real-time optimisation (RTO). ${ }^{1}$ In a large-scale plant the implementation of RTO has proved to be profitable. ${ }^{2,3,4}$ RTO drives operating condition towards the actual plant optimum in spite of model mismatch by adjusting selected optimization variables using measurement data. Problems face in RTO arise due to the inability to develop and adopt accurate models and the following are three different strategies which have been classified on how measurements are used to compensate the model uncertainty: model-parameter adaptation, modifier adaptation and input adaptation. ${ }^{5}$ All of these strategies have certain drawbacks and limitations. In particular, the modelparameter works well if the model structure is correctly developed and also reliably estimate the parameters from the available data. While, the modifier adaptation scheme can handle considerable plant-model mismatch by applying empirical bias and gradient-corrections to the objective and constraint functions in the iterative optimization procedure. In the case of the third strategies, direct input adaptation turns the optimization problem into a feedback control problem and implements optimality via tracking of appropriate control variables. The scheme achieves optimality despite the presence of uncertainty by using plant measurements but in practice to estimate the process gradients from noisy measurement data is one of the major challenges. ${ }^{6,7}$ In this study, we explored a new scheme for the modifier adaptation strategy that employs a better efficient process model constructed from ANN. ANN offers a new way of modelling nonlinear, uncertain and unknown complex system without requiring any explicit knowledge about the input-output relationship. It has more attractive advantages in which it can approximate any continuous or nonlinear function by using certain network configuration. ${ }^{8}$ ANN is widely accepted as a technique that is able to deal with non-linear problem, 


\section{Chemical Engineering Research Bulletin 19(2017) 129-138}

and once trained can perform prediction and generalization at high speed. ${ }^{9}$ Some of the recent studies carried out on the application of ANN models with the RTO strategies have demonstrated the potential of these strategies in determine optimal operating conditions for large-scale processes. ${ }^{10,11,12}$

The objective of this study is to explore the effect of the neural network process model application on the modified two step (MTS) technique ${ }^{13}$ for overcoming the problem of plant-model mismatch. The MTS is a control algorithm that employs the adaptive modifier scheme for determining the optimal control set-points. It also belongs to a class of algorithm known as Integrated System Optimisation and Parameter Estimation (ISOPE). This algorithm has attractive features compared to other algorithms in which it is able to generate a series of set-points converging to the real plant optimal solution in spite of uncertainty in the process model and disturbance estimates. ${ }^{14}$ The proposed new version of MTS technique will be using process model based on ANN which will be known as Iterative Neural Network Modified Two Step (INNMTS) technique

\section{Materials and Methods}

The RTO is a model based upper-level optimization system that is operated iteratively in closed loop and provides set-points to the lower-level regulatory control system in order to maintain the process operation as close as possible to the economic optimum. $^{7,15}$ In this section, 2CSTHEs as depicted in Figure 1, and it is used as a case study to test the performance of the Iterative Neural Network Modified Two Step (INNMTS) technique. ${ }^{16}$ Figure 1 also shows the feedback control configuration for the 2CSTHEs process at regulating level. The feedback control objectives of the 2CSTHEs control systems are to maintain the temperature and level of the fluid in both tanks at their targeted values.Table 1 shows the variables used in the modeling equations of 2CSTHEs process.

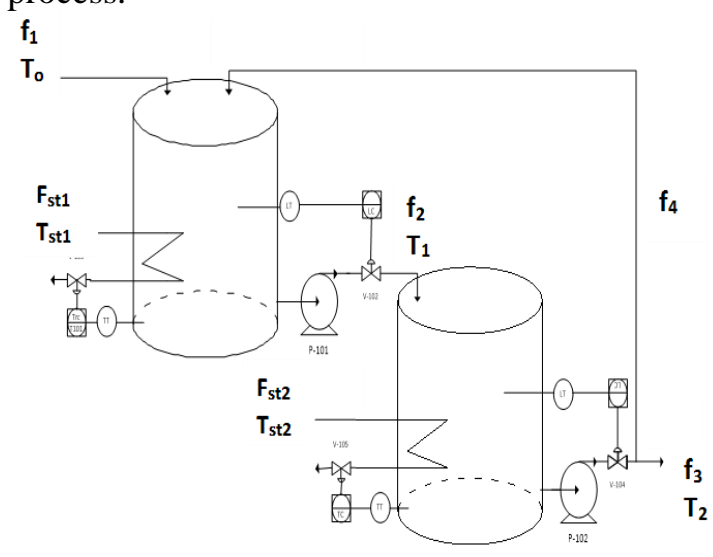

Figure 1: 2CSTHEs process layout with feedback control configuration.
Table 1: Variables used in the 2CSTHEs system.

\begin{tabular}{|c|c|c|c|}
\hline \multicolumn{2}{|c|}{ Variables } & Description & Unit \\
\hline Tank 1 & Tank 2 & & \\
\hline $\mathrm{f}_{1}$ & $\mathrm{f}_{2}$ & Flowrate input & $\mathrm{m}^{3} / \mathrm{min}$ \\
\hline $\mathrm{f}_{2}$ & $\mathrm{f}_{3}$ & Flowrate output & $\mathrm{m}^{3} / \mathrm{min}$ \\
\hline $\mathrm{T}_{0}$ & $\mathrm{~T}_{1}$ & Input temperature & ${ }^{0} \mathrm{C}$ \\
\hline & $\mathrm{f}_{4}$ & Recycle stream & $\mathrm{m}^{3} / \mathrm{min}$ \\
\hline & $\mathrm{T}_{2}$ & Outlet temperature & ${ }^{0} \mathrm{C}$ \\
\hline $\mathrm{h}_{1}$ & $\mathrm{~h}_{2}$ & Water level & $\mathrm{m}$ \\
\hline $\mathrm{f}_{\mathrm{st} 1}$ & $\mathrm{f}_{\mathrm{st} 2}$ & Steam Flowrate & $\mathrm{m}^{3} / \mathrm{min}$ \\
\hline $\mathrm{T}_{\mathrm{st} 1}$ & $\mathrm{~T}_{\mathrm{st}}$ & Steam temperature & ${ }^{0} \mathrm{C}$ \\
\hline $\mathrm{D}_{1}$ & $\mathrm{D}_{2}$ & Tank diameter & $\mathrm{m}$ \\
\hline $\mathrm{A}_{1}$ & $\mathrm{~A}_{2}$ & Tank area & $\mathrm{m}^{2}$ \\
\hline $\mathrm{A}_{\mathrm{C} 1}$ & $\mathrm{~A}_{\mathrm{C} 2}$ & Coil surface area & $\mathrm{m}^{2}$ \\
\hline
\end{tabular}

The dynamic mathematical models of the 2CSTHEs based on the conservation of heat and mass principle are as follows:

$A_{1} \frac{d h_{1}}{d t}=f_{1}+f_{4}-f_{2}$

$$
\begin{aligned}
& A h_{1} \frac{d T_{1}}{d t}=f_{1}\left(T_{0}-T_{1}\right)+f_{4}\left(T_{2}-T_{1}\right) \\
& +\frac{U A_{c 1}\left(T_{\mathrm{st} 1}-T_{1}\right)}{c_{p} \rho} \\
& C_{M} \frac{d T_{s t 1}}{d t}=\lambda w_{1}-U A_{c 1}\left(T_{s t 1}-T_{1}\right) \\
& A_{2} \frac{d h_{2}}{d t}=f_{2}-1.2 f_{3} \\
& A h_{2} \frac{d T_{2}}{d t}=f_{2}\left(T_{1}-T_{2}\right)+\frac{U A_{c 2}\left(T_{s t 2}-T_{2}\right)}{c_{p} \rho} \\
& C_{M} \frac{d T_{s t 2}}{d t}=\lambda w_{2}-U A_{c 2}\left(T_{s t 2}-T_{2}\right)
\end{aligned}
$$

The following are steady-state equations of the 2CSTHEs which have been adjusted to form the mathematical expression suitable to be used in the Optimizing Control Problem (OCP) in the supervisory control layer at RTO:

$$
\begin{aligned}
& f_{2}=C_{v} \sqrt{\rho g h_{1}} \\
& T_{s t 1}=\frac{f_{1}\left(T_{1}-T_{0}\right)+f_{4}\left(T_{1}-T_{2}\right)+\sigma_{1} T_{1}}{\sigma_{1}}
\end{aligned}
$$




\section{Chemical Engineering Research Bulletin 19(2017) 129-138}

$$
\begin{aligned}
& w_{1}=\frac{U A_{c 1}\left(T_{s t 1}-T_{1}\right)}{\lambda} \\
& f_{3}=C_{v} \sqrt{\rho g h_{2}} \\
& T_{s t 2}=\frac{f_{2}\left(T_{2}-T_{1}\right)+\sigma_{2} T_{2}}{\sigma_{2}} \\
& w_{2}=\frac{U A_{c 2}\left(T_{s t 2}-T_{2}\right)}{\lambda}
\end{aligned}
$$

Where

$\sigma_{1}=\frac{U A}{\rho c_{p} 1}$ and $\sigma_{2}=\frac{U A}{\rho c_{p}}$ The input and output data was generated from the steady-state open loop model of the 2CSTHEs as shown in Figure 2 which is the Simulink data generation block for 2CSTHEs process. Data generation is implemented using the MATLAB Simulink software. The parameter values used in the simulation are shown in Table 2.

Table 2: Values of parameters and constants used in data generating.

\begin{tabular}{|l|c|c|}
\hline Variables & Value & Unit \\
\hline $\mathrm{T}_{0}$ & 30 & ${ }^{0} \mathrm{C}$ \\
\hline $\mathrm{f}_{1}$ & 0.012 & $\mathrm{~m}^{3} / \mathrm{min}$ \\
\hline $\mathrm{f}_{2}$ & 0.015 & $\mathrm{~m}^{3} / \mathrm{min}$ \\
\hline $\mathrm{A}_{1}$ & 1.767 & $\mathrm{~m}^{2}$ \\
\hline $\mathrm{A}_{2}$ & 1.767 & $\mathrm{~m}^{2}$ \\
\hline $\mathrm{g}$ & 9.8 & $\mathrm{~m} / \mathrm{s}^{2}$ \\
\hline $\mathrm{C}_{\mathrm{M}}$ & 0.504 & $\mathrm{~kJ} / \mathrm{kg} \cdot \mathrm{K}$ \\
\hline $\mathrm{Cp}$ & 4.187 & $\mathrm{~kJ} / \mathrm{kg} \cdot \mathrm{K}$ \\
\hline $\mathrm{UAc} 1$ & 12.52 & $\mathrm{~kJ} / \mathrm{kg} \cdot \mathrm{m}^{3}$ \\
\hline $\mathrm{UAc} 2$ & 98.21 & $\mathrm{~kJ} / \mathrm{kg} \cdot \mathrm{m}^{3}$ \\
\hline$\rho$ & 1000 & $\mathrm{~kg} / \mathrm{m}^{3}$ \\
\hline
\end{tabular}

Simulink block diagrams of the open loop process models for tank 1 and 2 of the 2CSTHEs are built according to the Eqs. (7) to (12) and they are as shown in Figures 3 and 4, respectively.

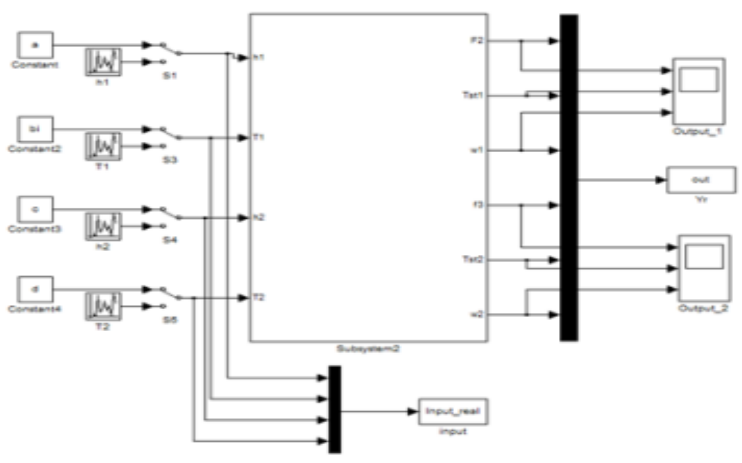

Figure 2: Simulink data generation block of 2CSTHE.

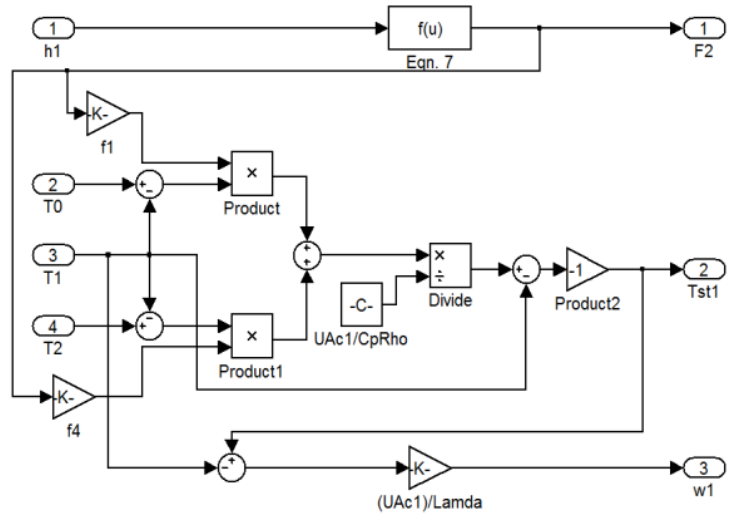

Figure 3: Simulink Model of tank 1 of 2CSTHEs.

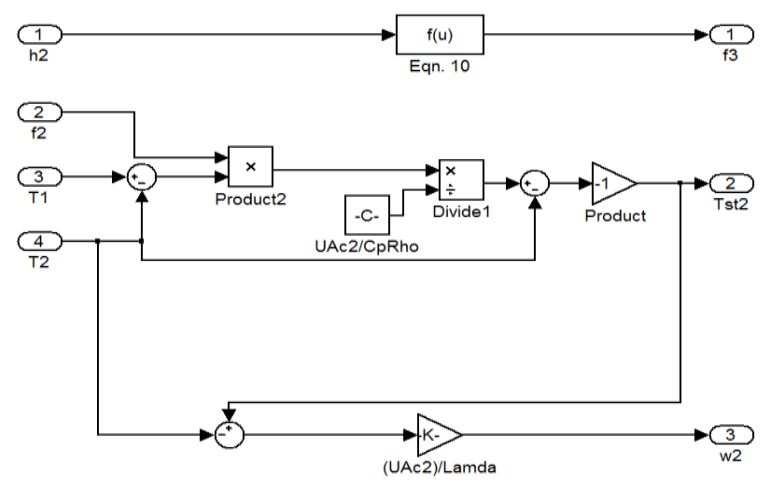

Figure 4: Simulink Model of tank 2 of 2CSTHEs.

The data generating block was run to generate the process data. Data collected is taken as real process data, and is used for system identification of the 2CSTHEs, where the data is supplied in the training of the network. In the simulation, 5000 data sets were generated for the 2CSTHEs system at steady-state condition.

\section{Neural network model of the 2CSTHEs}

Neurons are processing units in an ANN where a set of neurons grouped together in layers which inter-relate with others by parameter called weights and these weights are used to model complex relationships between inputs and outputs by adjusted their values. The neural network consists of three layers: The first layer is the inlet layer which receives input data, the second layer may made up of one or more layers known as 'hidden' layer and the third layer is the output layer which propagates the information from network back to the outside as predicted output. Each neuron in the hidden layer carries out a weighted addition of its inputs and then applies its transfer function to pass on the information from the input layer to the hidden layer. The response from the neurons in the hidden layer acts as input to the neurons in the output layer, producing the weighted sum and applying another transfer function. ${ }^{17}$ 


\section{Chemical Engineering Research Bulletin 19(2017) 129-138}

Figure 5 shows a typical three layered neural network structurewhere $y(k)$ is the final output of the network, $X_{(r)}$ is input value of the network, $J$ is the neuron number in the hidden layer, $I W$ are the weights in the input-hidden layer, $b l$ are the biases in the hidden layer.

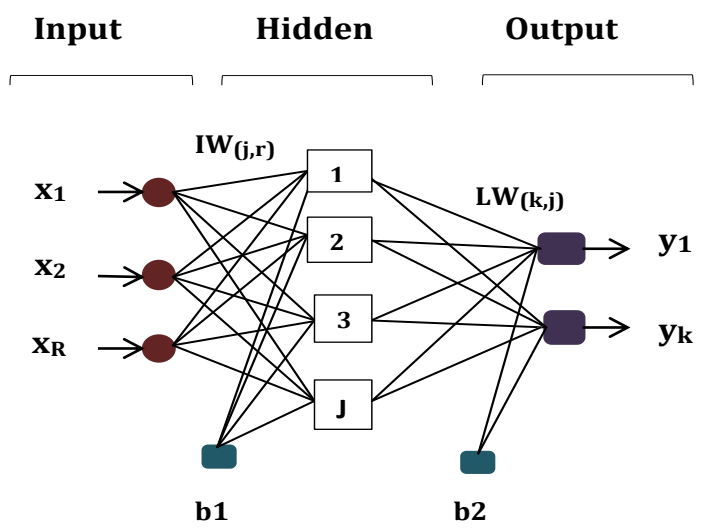

Figure 5: A typical three layered neural network structure.

$L W$ are the weights in the hidden output layer, $R$ is the input-neuron number and $b 2$ is the biases in the output layer. Two types of neural network models were considered to predict the output with respect to the generated data, the first type is where the hidden layers applied differentiable transfer function in the form of the hyperbolic tangent sigmoid (TANSIG) and linear transfer function (PURELIN) was used in the output layer and whilst in the second type the linear transfer function was used for both hidden layer and output layer to predict the outputs with respect to the generated data. A general neural network model with $F_{t}(X)$ in the hidden layer and $F_{p}(X)$ in the output layer is given by Eq. 13:

$$
\begin{array}{r}
y_{(k)}=F_{p}(X)\left(\sum _ { j = 1 } ^ { J } \left[L W _ { ( k , j ) } \left(F _ { t } ( X ) \sum _ { r = 1 } ^ { R } \left(I W_{(j, r)} X_{(r)}\right.\right.\right.\right. \\
\left.\left.\left.+b 1_{(j)}\right)+b 2_{(k)}\right]\right)
\end{array}
$$

where $F(X)$ is the transfer function that is used to the normalized output values from the neurons. These TANSIG and PURELIN functions can be defined as $F(X)=\frac{e^{X}-e^{-X}}{e^{X}+e^{-X}}$ and $F(X)=X$, respectively. The ANN models for the 2CSTHEs at steady-state were developed using the multilayer feed-forward network or MLFFN. In this study the second NN model of 2CSTHEs, which in the linear form, was chosen to be applied to the proposed INNMTS algorithm at the RTO level because it is more favorable in the prediction of 2CSTHEs outputs. ${ }^{18}$ The training of MLFFN, a number of parameters of the network need to be determined, like the number of hidden layers in the network, number of neurons in the hidden layer, and distribution of data between training, validation, and testing of network. In the study, the main methods used to determine the performance of the ANN models are the mean squared error, MSE and coefficient of determination, $\mathrm{R}^{2}$, and they were calculated as follows ${ }^{19}$ :

$$
\begin{gathered}
\mathrm{MSE}=\frac{\sum_{i=1}^{n}\left(y_{i}^{e}-y_{i}^{p}\right)^{2}}{n} \\
\mathrm{R}^{2}=1-n \frac{\sum_{i=1}^{n}\left(y^{e}-y_{i}^{p}\right)^{2}}{\sum_{i=1}^{n}\left(y^{e}-y_{i}^{e}\right)^{2}}
\end{gathered}
$$

where

$$
\begin{aligned}
& \bar{y}_{i}^{e} \text { the average experimental value } \\
& y_{i}^{p} \text { the predicted value, } \\
& y_{i}^{e} \text { the experimental value, } \\
& n \text { the number of experiments. }
\end{aligned}
$$

The best performance of the ANN architecture is determined by the lowest MSE and the maximum coefficient of determination $R^{2}$ that defines the integrity of the experimental data. ${ }^{20}$ In the training of neural network the Levenberg-Marquardt backpropagation (LM) training algorithm was used. For the training algorithm, optimum structure of ANN model is determined, according to the lowest MSE value. The network training was carried out using the MATLAB Neural Network Toolbox.

\section{Optimizing control problem of 2CSTHEs}

The Optimization Control Problem (OCP) for the process plant can be mathematically formulated as:

$$
\begin{aligned}
& \min _{c} Q(c, y) \\
& \\
& y=F_{*}(c) \\
& \stackrel{\Delta}{(c, y) \in C Y \stackrel{1}{=}\{(c, y) \leq 0\}}
\end{aligned}
$$

where

$c=\left[c_{1}, c_{2}, ., c_{n}\right]^{T} \in R^{n} \quad$ process control vectors 


\section{Chemical Engineering Research Bulletin 19(2017) 129-138}

$y=\left[y_{1}, y_{2}, ., y_{n}\right]^{T} \in R^{m} \quad$ output vectors

$Q: R^{n} \mathrm{x} R^{m} \rightarrow R$ is a given performance map,

$F_{*}: R^{n} \rightarrow R^{m}$ is the real process input-output map and $G: R^{n} x R^{m} \rightarrow R^{p}$ is the process inequality constraint map. The maps $\mathrm{Q}$ and $\mathrm{G}$ are assumed to be known exactly.Introducing a process model and formulate another optimization problem (OCP1):

$$
\begin{array}{ll}
\min _{c, \alpha} q(c, \alpha) & \\
\text { s.t } & F_{N}(c, \alpha)=F_{*}(c) \\
& g(c, \alpha) \leq 0
\end{array}
$$

where $g(c, \alpha) \stackrel{\Delta}{=} G\left(c, F_{N}(c, \alpha)\right), \stackrel{\Delta}{q=} Q\left(c, F_{N}(c, \alpha)\right)$

$F_{N}: R^{n} x R^{m} \rightarrow R^{m}$ is the model input-output mapping which is only an approximation to real output process and $\alpha$ is the model parameter vector. It is assumed that the map $F_{*}($.$) is not known exactly. The$ 2CSTHEs is a process consists of two subsystems which can be depicted in the block diagram as shown in Figure 6.

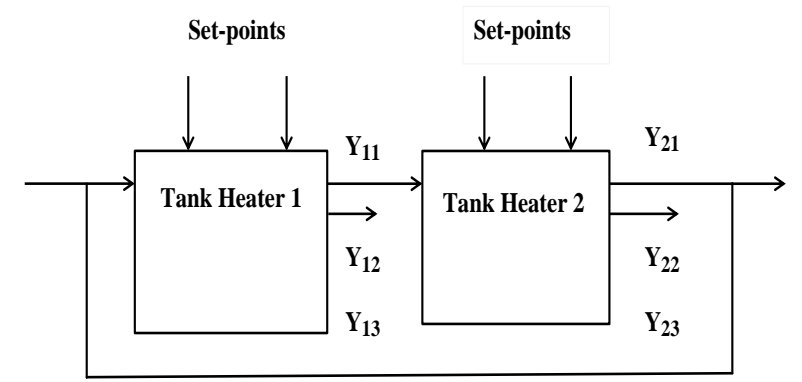

Figure 6: The sub-system block diagram for 2CSTHEs.

The Optimizing Control Problem (OCP) for the 2CSTHEs can be described as follows:

The objective function:

$$
\begin{aligned}
& Q=3 \times 10^{-4}\left(1767\left(c_{11}+c_{21}\right)\right) \\
& +6.45 \times 10^{-6}\left[1179.52\left(y_{12}-c_{21}\right)\left(y_{22}-c_{12}\right)\right]
\end{aligned}
$$

The real process output:

$$
y_{*}=A^{-1} B_{1}
$$

where

$$
y_{*}=\left[\begin{array}{c}
y_{* 11} \\
y_{* 12} \\
y_{* 13} \\
y_{* 21} \\
y_{* 22} \\
y_{* 23}
\end{array}\right]
$$

$$
A_{1}=\left[\begin{array}{ccccc}
1 & 0 & 0 & 0 & 0 \\
24+0.2 c_{22}-c_{21} & 0 & 0 & 0 & 0 \\
0 & 12.5 & -2260 & 0 & 0 \\
0 & 0 & 0 & 1 & 0 \\
c_{21}-c_{22} & 0 & 0 & 0 & 0 \\
0 & 0 & 0 & 94 & -2260
\end{array}\right]
$$

$$
B_{1}=\left[\begin{array}{c}
0.014 \sqrt{c_{11}} \\
0.003 c_{21} \\
12.5 c_{21} \\
0.014 \sqrt{c_{12}} \\
0.023 c_{22} \\
94.21 c_{22}
\end{array}\right]
$$

The neural network output process model:

$$
y_{N}=A_{2}+B_{2}
$$

$$
y_{N}=\left[\begin{array}{l}
y_{N 11} \\
y_{N 12} \\
y_{N 13} \\
y_{N 21} \\
y_{N 22} \\
y_{N 23}
\end{array}\right]
$$

$$
A_{2}=\left[\begin{array}{l}
\sum_{j=1}^{J}\left[L W_{(1, j)}\left(R \sum_{r=1}^{R} I W_{(j, r)} X_{(r)}+b 1_{(j)}\right)\right]+b 2_{(1)} \\
\sum_{j=1}^{J}\left[L W_{(2, j)}\left(R \sum_{r=1}^{R} I W_{(j, r)} X_{(r)}+b 1_{(j)}\right)\right]+b 2_{(2)} \\
\sum_{j=1}^{J}\left[L W_{(3, j)}\left(R \sum_{r=1}^{R} I W_{(j, r)} X_{(r)}+b 1_{(j)}\right)\right]+b 2_{(3)} \\
\sum_{j=1}^{J}\left[L W_{(4, j)}\left(R \sum_{r=1}^{R} I W_{(j, r)} X_{(r)}+b 1_{(j)}\right)\right]+b 2_{(4)} \\
\sum_{j=1}^{J}\left[L W_{(5, j)}\left(R \sum_{r=1}^{R} I W_{(j, r)} X_{(r)}+b 1_{(j)}\right)\right]+b 2_{(5)} \\
\sum_{j=1}^{J}\left[L W_{(6, j)}\left(R \sum_{r=1}^{R} I W_{(j, r)} X_{(r)}+b 1_{(j)}\right)\right]+b 2_{(6)}
\end{array}\right]
$$




\section{Chemical Engineering Research Bulletin 19(2017) 129-138}

where $X_{(r)}$ is the input into the network, $\mathrm{R}$ is the number of input variables and $\mathrm{J}$ is the neuron number in the hidden layer. The equivalent representation of $y_{i j}, c_{i j}$ and $X_{(r)}$ to the variables in the 2CSTHEs are shown in Table 3.

Table 3: Variables representation.

\begin{tabular}{|l|c|c|}
\hline \multicolumn{1}{|c|}{ Parameter } & Symbol & Represent \\
\hline $\begin{array}{l}\text { Output flow rate from tank } \\
1\end{array}$ & $\mathrm{f}_{2}$ & $\mathrm{y}_{11}$ \\
\hline $\begin{array}{l}\text { Output temperature from } \\
\text { tank 1 }\end{array}$ & $\mathrm{T}_{\mathrm{st1}}$ & $\mathrm{y}_{12}$ \\
\hline $\begin{array}{l}\text { Steam flow rate from tank } \\
1\end{array}$ & $\mathrm{w}_{1}$ & $\mathrm{y}_{13}$ \\
\hline $\begin{array}{l}\text { Output flow rate from tank } \\
2\end{array}$ & $\mathrm{f}_{3}$ & $\mathrm{y}_{21}$ \\
\hline $\begin{array}{l}\text { Temperature output from } \\
\text { tank 2 }\end{array}$ & $\mathrm{T}_{\mathrm{st} 2}$ & $\mathrm{y}_{22}$ \\
\hline $\begin{array}{l}\text { Steam flow rate from tank } \\
2\end{array}$ & $\mathrm{w}_{2}$ & $\mathrm{y}_{23}$ \\
\hline Height of tank 1 & $\mathrm{h}_{1,}$ & $\mathrm{c}_{11}, \mathrm{X}_{1}$ \\
\hline Height of tank 2 & $\mathrm{h}_{2}$ & $\mathrm{c}_{21}, \mathrm{X}_{2}$ \\
\hline Temperature of tank 1 & $\mathrm{T}_{1}$ & $\mathrm{c}_{12}, \mathrm{X}_{3}$ \\
\hline Temperature of tank 1 & $\mathrm{T}_{2}$, & $\mathrm{c}_{22}, \mathrm{X}_{4}$ \\
\hline
\end{tabular}

Parameter estimation:

$$
B_{2}=\left[\begin{array}{l}
\alpha_{11} \\
\alpha_{12} \\
\alpha_{13} \\
\alpha_{21} \\
\alpha_{22} \\
\alpha_{23}
\end{array}\right]
$$

The parameter estimations are added to NN model of the process for the purpose of performing parameter estimation task which takes in to account the modelplant miss-match. The values of the parameters that used in optimization are tabulated in Table 4.
Table 4: Value of parameter that used in optimization.

\begin{tabular}{|c|c|}
\hline Parameter & Value \\
\hline Water cost, $\$_{\mathrm{W}}(\mathrm{RM} / \mathrm{kg})$ & $2.07 \times 10^{-3}$ \\
\hline Steam cost, $\$_{\mathrm{s}}(\mathrm{RM} / \mathrm{kJ})$ & $2.79 \times 10^{-5}$ \\
\hline
\end{tabular}

Source: Jabatan Bekalan Air (JBA) - Water Malaysia 2015, Hamada boiler 2008.

\section{INNMTS technique}

In this study, a new version of MTS algorithm is developed by integrating MTS technique with neural network process model (Eq. 21) which will be known as INNMTS technique. The INNMTS is applied in the RTO control level to recalculate the optimum steadystate set points for obtaining the current optimum operating conditions for the 2CSTHEs process. The proposed algorithm can be implemented as follows, for given initial control set-point $v^{0} \in C$ and the initial estimate for $\xi^{0}$ :

i. Enter $v^{k}$ to the actual process and the output of $F_{*}\left(v^{k}\right)$ measured. Determine $\alpha^{k}$ by solving:

$$
F_{N}\left(v^{k}, \alpha\right)=F_{*}\left(v^{k}\right)
$$

Perform additional perturbations about $v^{k}$ and measure the corresponding process output and compute finite difference approximation of $\frac{\partial^{T} F_{*}(v)}{\partial v}$. Calculate $\lambda^{k}=\lambda\left({ }^{\wedge-1}, v^{k}, \alpha^{k}, \xi^{k}\right)$, where:

$$
\begin{aligned}
& \lambda(c, v, \alpha, \xi)=\left[\frac{\partial^{T} F_{N}(v, \alpha)}{\partial v}-\frac{\partial^{T} F_{*}(v)}{\partial v}\right]\left[\frac{\partial^{T} F_{N}(v, \alpha)}{\partial v}\right]^{-1} . \\
& .\left[\frac{\partial^{T} Q\left(c, F_{N}(v, \alpha)\right)}{\partial \alpha}+\frac{\partial^{T} G\left(c, F_{N}(c, \alpha)\right)}{\partial \alpha} \xi\right]
\end{aligned}
$$

ii. Solve the modified optimization problem (Eq. 24) to $\wedge k$

obtain $c$ and associated Langrange multipliers, $\wedge k$ $\xi$.

$\min _{c} Q\left(c, F_{N}(c, \alpha)\right)-\lambda^{T} c+0.5 \rho\|c-v\|^{2}$

s.t $\quad g(c, \alpha) \leq 0$ 


\section{Chemical Engineering Research Bulletin 19(2017) 129-138}

If $\hat{c}^{k}=v^{k}$ and $\hat{\xi}^{k}=\xi^{k}$ the iteration is terminated. Otherwise, updates $v^{k}$ and $\xi^{k}$ as follows:

$$
\begin{gathered}
v^{k+1}=v^{k}+\varepsilon_{v}\left(c^{k}-v^{k}\right) \\
\xi^{k+1}=\xi^{k}+\varepsilon_{\xi^{k}}\left(\xi^{k}+\xi^{k}\right)
\end{gathered}
$$

\section{Results and Discussion}

Extensive simulation studies have been performed on the 2CSTHEs for evaluating the effectiveness of the proposed INNMTS algorithm. The NN model of 2CSTHEs was developed from a set of 5,000 of inputoutput data of the process generated. The ANN model of 2CSTHEs is trained using $h_{1}, h_{2}, T_{1}$, and $T_{2}$ as inputs into the network to predict $f_{2}, T_{s t 1}, w_{1}, f_{3}, T_{s t 2}$ and $w_{2}$ as the outputs from the network. The optimal and stable data distribution was determined to be when using $85 \%$ of the data for training, $10 \%$ for validation and 5\% for testing.

The data distribution obtained is trained using Levenberg-Marquardt (LM) training function to obtain the optimal NN structure. Linear transfer function is used since it more favorable in predicting output of the new random input. During training, the weights and biases are changed in order to minimize the error between the outputs values and the data.The training process is carried out by comparing the output from the network to the target outputs from the given data.Figure 7 shows the neural network performance for the MSE value between training, validation and testing data for 2CSTHEs.

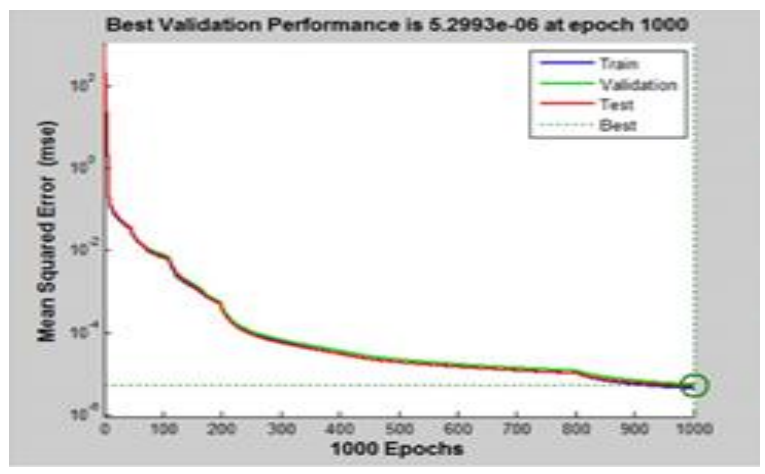

Figure 7: The MSE between training, validation and testing for 2CSTHEs.
Figure 8 shows the coefficient of determination $R^{2}$ between target value and output value for the 2CSTHEs. An overall value $R^{2}$ equal to one indicates adequacy of the model proposed for the prediction of the behavior of 2CSTHEs system.

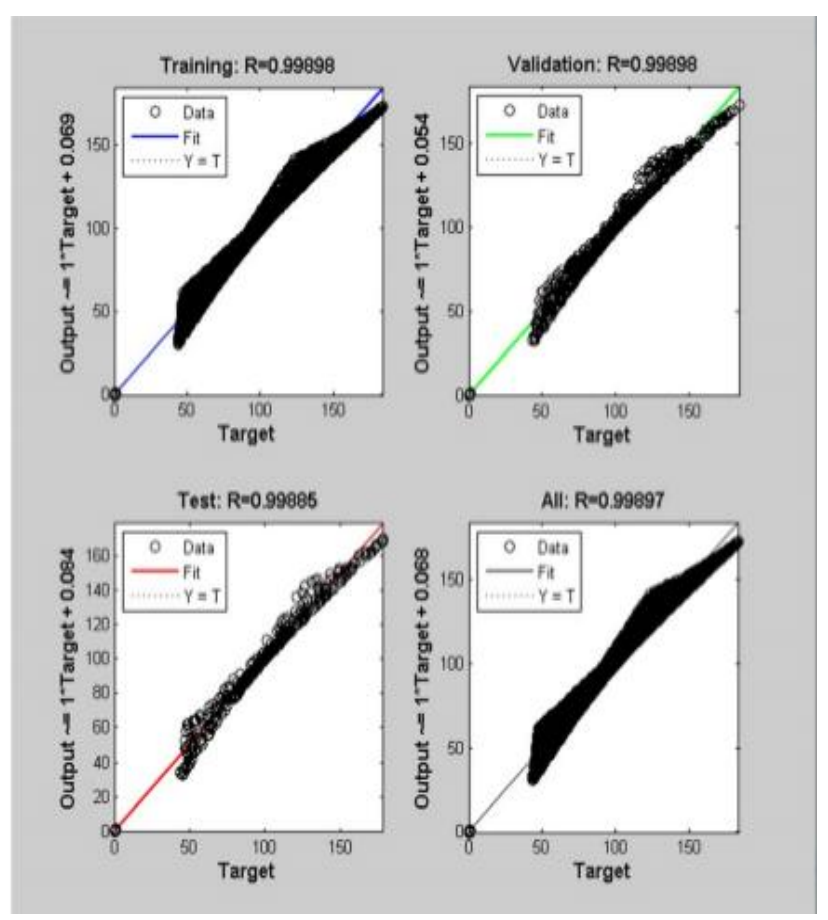

Figure 8: $\mathrm{R}^{2}$ between target value and output for 2CSTHEs.

Figure 9 shows the optimal ANN configuration obtained for the linear model with MLFFN (4-10-6) structure. The best fitting training data acquired with optimal network structure is integrated into INNMTS to be used to determine the optimal set-points of 2 CSTHEs by minimizing the OCP1 of the 2CSTHEs process.

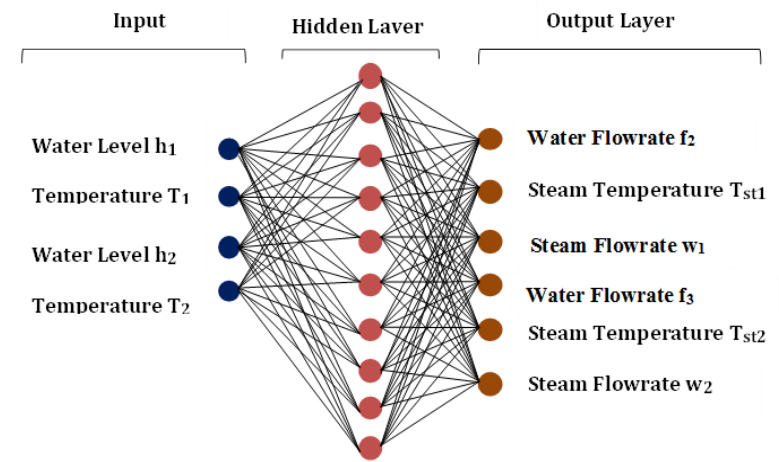

Figure 9: Diagram of optimal ANN configuration

The real optimal solutions of 2CSTHEs as shown in Table 5, were determined in order to determine the effectiveness of the proposed algorithm. The optimal 


\section{Chemical Engineering Research Bulletin 19(2017) 129-138}

values for $c_{12}$ and $c_{22}$ are obtained. While keeping the variables $c_{11}$ and $c_{21}$ which represented by $h_{1}$ and $h_{2}$ respectively, at constant values of $1.5 \mathrm{~m}$.

Table 5: The real optimal solutions of 2CSTHEs.

\begin{tabular}{|c|c|}
\hline Parameters & Value \\
\hline$c_{11}$ & $1.5 \mathrm{~m}$ \\
\hline $\mathrm{c}_{12}$ & $1.5 \mathrm{~m}$ \\
\hline $\mathrm{c}_{21}$ & $50^{0} \mathrm{C}$ \\
\hline $\mathrm{c}_{22}$ & $80^{\circ} \mathrm{C}$ \\
\hline Real Objective function & 11.0535 \\
\hline
\end{tabular}

The INNMTS technique was simulated on 2CSTHEs with error tolerance of 0.0005 and under various conditions where step change $\left(\varepsilon_{v}\right)$ varies from values of 0.1 to 0.9 and penalty coefficient from values of 0 to 1. Table 6 shows the simulation results of iteration number versus step change coupled with penalty coefficient obtained for the INNMTS technique.The simulation results show that the least number of iteration is obtained with step change at 0.6 and penalty coefficient values from 0 to 0.5 .

Table 6: INNMTS technique with linear function results for 2CSTHEs.

\begin{tabular}{|c|c|c|c|c|c|c|c|}
\hline \multirow{2}{*}{$\varepsilon_{v}$} & \multicolumn{7}{|c}{$\rho$} \\
& 0 & 0.1 & 0.2 & 0.3 & 0.4 & 0.5 & \\
\hline 0.1 & - & - & - & - & - & - & - \\
\hline 0.2 & 46 & 46 & 46 & 46 & 46 & 46 & 11.0648 \\
\hline 0.3 & - & 34 & 30 & 50 & 63 & 93 & 0.0000 \\
\hline 0.4 & 21 & 21 & 21 & 21 & 21 & 21 & 11.0650 \\
\hline 0.5 & - & - & - & - & - & - & - \\
\hline 0.6 & 5 & 5 & 5 & 5 & 5 & 5 & 11.0550 \\
\hline 0.7 & 10 & 10 & 10 & 10 & 10 & 10 & 11.0657 \\
\hline 0.8 & 8 & 8 & 8 & 8 & 8 & 8 & 11.0660 \\
\hline 0.9 & 6 & 6 & 6 & 6 & 6 & 6 & 11.0661 \\
\hline 1.0 & 2 & 2 & 2 & 2 & 2 & 2 & 11.0665 \\
\hline
\end{tabular}

Result from Table 7 also indicates that the objective function obtained is also the most closest to the real objective function value which required 5 iterations only to get the optimum set-points which about one third less compared to that of the MTS technique which converges to optimum set-points with 16 iterations. ${ }^{18}$ INNMTS was able to reduce number of iterations, because the $\mathrm{NN}$ model output equations employed in INNMTS have much better accuracy compared to the model output equations used in MTS technique which based on trial and error basis.
Table 7: Comparison for MTS and INNMTS techniques.

\begin{tabular}{|l|c|c|}
\hline \multicolumn{1}{|c|}{ Parameter } & MTS & INNMTS \\
\hline Suitable step value & 0.5 & 0.6 \\
\hline Final objective function & 11.0535 & 11.0539 \\
\hline Accuracy optimization & $0.0018 \%$ & $0.0018 \%$ \\
\hline Iteration needed & 16 & 5 \\
\hline
\end{tabular}

Figure 9 shows the values of the objective functions verses iteration numbers for both MTS and INNMTS during the simulations period. Figure 10 shows the movement of set points toward the optimum set point values verses the number of iterations. As the iteration number progresses, the two set-points $c_{12}$ and $c_{22}$ whichare moving slowly toward the optimum values and lastly it stopped when the tolerance error for the optimum set points has been achieved

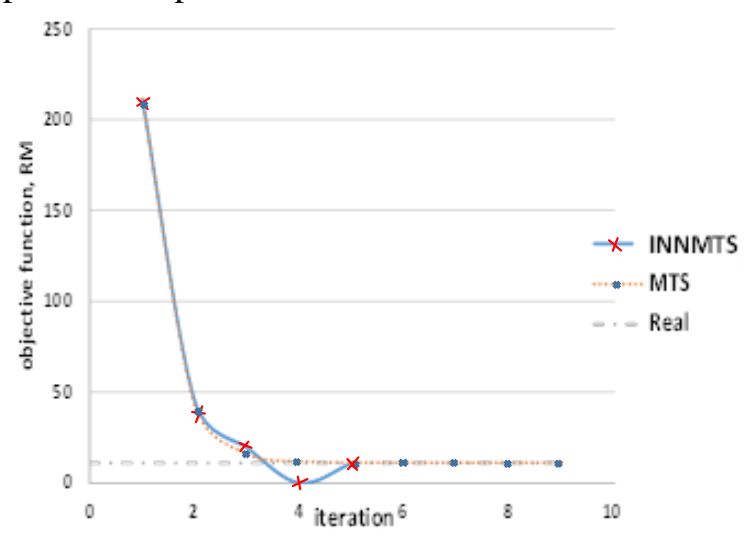

Figure 9: Value of objective function versus iteration number.

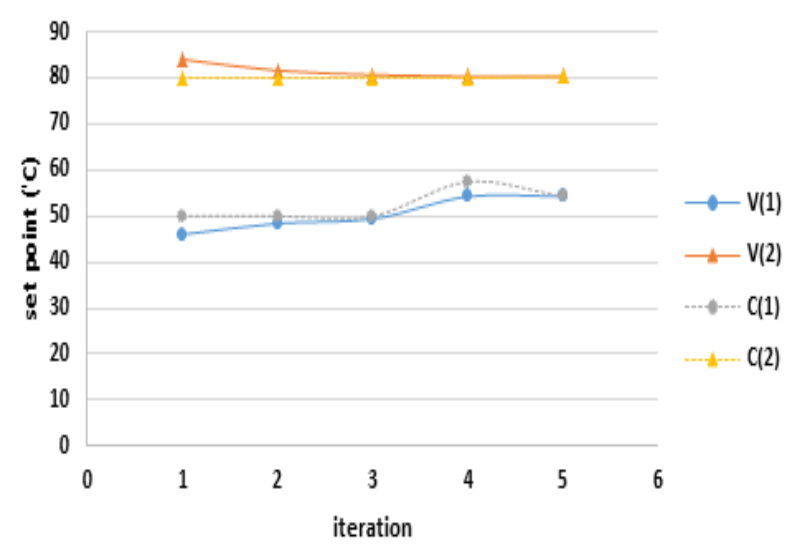

Figure 10: Set point values versus the number of iterations. 


\section{Chemical Engineering Research Bulletin 19(2017) 129-138}

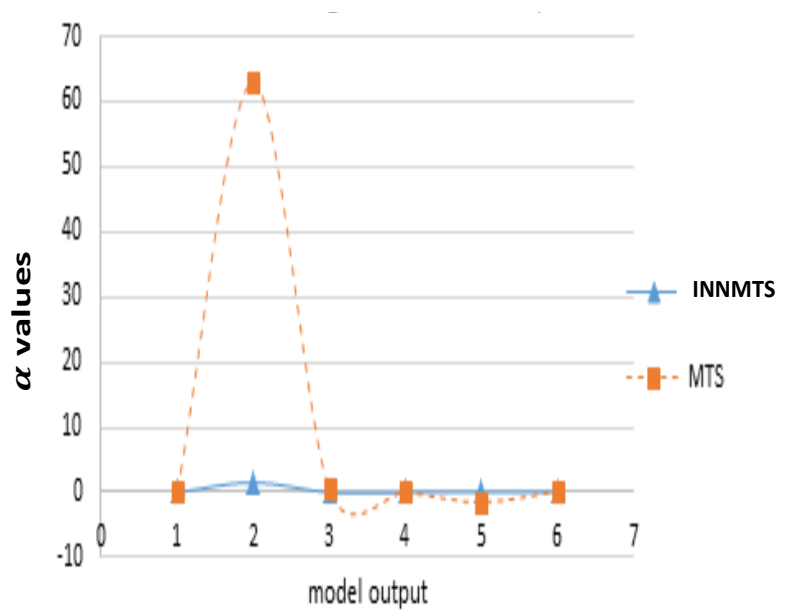

Figure 11: Values of parameter estimation, $\alpha$, for two of the output models.

Figure 11 shows that the two-parameter estimation values for INNMTS are significantly less than that of MTS, and which indicates that the model-plant mismatch is greatly reduced in INNMTS compared to MTS which explained the reason why the number of iterations required by INNMTS significantly less than that required by MTS technique.

\section{Conclusion}

Mathematical model for 2CSTHEs has been developed using the first principle which use to represent the real process plant. Simulink models for real process plant were constructed and used for generating input-output plant data. ANN model of the process was developed and trained using generated data and the most suitable ANN model structure determined is used in the optimization problem at the RTO. The multilayer feed forward network architecture 4-10-6 with linear function used in both the hidden and the ouput layers was found to be efficient enough to model the 2CSTHEs and used in the INNMST technique. Simulation results show that the INNMTS technique has successfully improved the performance of MTS by reducing the number of iteration required to one third and also able to reduce significantly the model-plant mismatch.

\section{Acknowledgement}

This research is supported by the Exploratory Research Grant Scheme (ERGS/1/2013/TK05/UKM/02/1) under the Ministry of Education Malaysia. The authors wish to express their thanks for the support.

\section{References}

1. D. E. Seborg, T. F. Edgar., D. A. Mellichamp and F. J. Doyle III, Process Dynamics and Control, (3rd ed.). USA: Wiley, 2011.
2. J. K. Bailey, N. Hrymak, S. S. Treiba andR. B. Hawkins, "Non-linear optimization of a hydrocracker fractionation plant," Computers and Chemical Engineering,vol. 17, pp. 123, 1993.

3. W. S. Yip and T. E. Marlin, "Designing plant experiments for real-time optimization systems," Control engineering Practice, vol. 11, pp. 837-845, 2003.

4. R. Mazaeda, S. P. Cristea and C. D. Prada, "Plantwide hierarchical optimal control of crystallization process," IFAC-PapersOnLine, vol. 48-8, pp. 1210-1215, 2015.

5. B. Chachuat, B. Srinivasan and R B. D. Bonvin, "Adaptation strategies for real-time optimization," Computers and Chemical Engineering,vol. 33, pp. 1557-1567, 2009.

6. 6.T. Rodriguez-Blanco, D. Sarabia, D. Navia and C. D. Prada, "Modifier-adaptation methodology for RTO applied to Distillation Columns," IFACPaperOnLine, vol. 48-8, pp. 223-228, 2015.

7. W. Gao, S. Wenzel and S. Engell, "A reliable modifier-adaptation strategy for real-time optimization," Computers and Chemical Engineering,vol. 91, pp. 318-328, 2016.

8. G. N. Xie, Q. W. Wang, M. Zeng and L. Q. Luo, "Heat transfer analysis for shell-and-tube heat exchangers with experimental data by artificial neural networks approach," Applied Thermal Engineering, vol. 27, pp. 1096-1104, 2007.

9. R. Mikulandric, D. Loncar, D. Bohning, R. Bohme and M. Beckmann, "Artificial neural network modelling approach for biomass gasification," Energy Conversion and Management, vol. 87, pp.1210-1223, 2014.

10. N. F. Osuolale and J. Zhang, "Energy efficiency optimisation for distillation column using artificial neural network models," Energy, vol. 106, pp.562578, 2016.

11. J. D. Reyes, A. I. Rodriguez and C. A. M. Riascos, "Data analysis and modelling of a fluid catalytic cracking unit (FCCU) for an implementation of real time optimization," $12^{\text {th }}$ International Symposium on Process System Engineering and $25^{\text {th }}$ European Symposium on Computer Aided Process Engineering. Copenhagen, Denmark, 2015.

12. M. Lawrynczuk, "On-line set-point and predictive control using neural Hammerstein models," Chemical Engineering Journal, vol. 166, pp. 269287, 2011.

13. P. D. Roberts, "An algorithm for steady-state system optimization and parameter estimation," Int. J. Syst. Sci., vol. 10, no. 7, pp. 719-734, 1979. 


\section{Chemical Engineering Research Bulletin 19(2017) 129-138}

14. P. Tatjewski, Advanced Control of Industrial Processes Structures and Algorithms, London, Springer-Verlag, 2007.

15. D. Gajic, I. Savic-Gajic, I. Savic and O. Georgieva, "Modelling of electrical energy consumption in an electric arc furnace using artificial neural networks," Energy, vol. 108, pp 132-139, 2016.

16. M. Y. Kiu, Optimal control design of two continuous stirred tank heaters is Series, Thesis, National University of Malaysia (UKM), Bangi, 2012.

17. E. M. Alvarez, J. A. Hernandez and M. Bourous, "Modelling the performance parameters of a horizontal falling film absorber with aqueous (lithium, potassium, sodium) nitrate solution using artificial neural networks," Energy, vol. 102, pp. 313-323, 2016.

18. M. A. Mohd Razali, Real-time optimizing using ISOPE technique integrate with neural network, Thesis, National University of Malaysia (UKM), Bangi, 2016.

19. W. Gao, S. Wenzel and S. Engell, "A reliable modifier-adaptation strategy for real-time optimization," Computers and Chemical Engineering,vol. 91, pp. 318-328, 2016.

20. C. I. Koon, K. I. Soo, C. T. Peh and H. Y. Kim, "Modeling electrostatic process using artificial neural network (ANN)," Procedia Computer Science, vol. 91, pp. 372-381, 2016.

\section{Available online at http://www.banglajol.info/index.php/CERB}

Publisher: Department of Chemical Engineering, Bangladesh University of Engineering and Technology (BUET). Review \& Publication: A submitted original manuscript is taken into review only if the uniqueness is found to be more than $85 \%$ in plag-scanning and selected for publication by the complete acceptance from at least two reviewers out of three. Home Page: http://www.banglajol.info/index.php/CERB . Indexed by Chemical Abstract Service (CAS), CEABA-VtB, Google Scholar, Scopus and DOAJ. 